Proceedings of the 16th Czech and Slovak Conference on Magnetism, Košice, Slovakia, June 13-17, 2016

\title{
Structure of Melt-Spun $\mathrm{Co}_{2} \mathrm{MnAl}$ Heusler Alloy
}

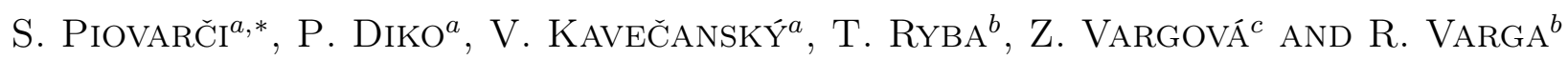 \\ ${ }^{a}$ Institute of Experimental Physics, Slovak Academy of Sciences, Watsonova 47, 04001 Košice, Slovakia \\ ${ }^{b}$ Institute of Physics, Faculty of Sciences, P.J. Šafárik University, Park Angelinum 9, 04001 Košice, Slovakia \\ ${ }^{c}$ Institute of Chemistry, Faculty of Sciences, P.J. Šafárik University, Moyzesova 11, 04001 Košice, Slovakia
}

\begin{abstract}
The growth-related microstructure and texture of the $\mathrm{Co}_{2} \mathrm{MnAl}$-type Heusler alloy in the form of a melt-spun ribbon was studied by electron microscopy, electron backscattered diffraction and X-ray diffraction. It is shown that melt spinning produces a single-phase disordered Heusler alloy. The fine grain structure at the wheel side of the ribbon exhibits no texture, while dominant columnar grain structure formed on the free surface side exhibits the $\langle 111\rangle$ fibre texture with a declination by about 10 degrees in the spinning direction. The dendritic growth of columnar crystals causes inhomogeneity of the chemical composition on a micrometre scale with a higher Co and $\mathrm{Al}$ concentration in the centre of dendritic arms and a higher concentration of $\mathrm{Mn}$ at the dendrite arm boundaries.
\end{abstract}

DOI: 10.12693/APhysPolA.131.881

PACS/topics: 68.55.-a, 75.50.Cc, 61.05.cp

\section{Introduction}

The Heusler alloys (HA) of the $\mathrm{Co}_{2} \mathrm{MnAl}$ type are studied for their high magnetic moment and relatively high spin polarization [1]. HA are usually prepared by arcmelting. After slow-cooling solidification, the cast samples have a multiphase structure; therefore, annealing is needed for sample homogenization. Later, a new method for HA preparation was introduced, which employs rapid quenching by melt spinning, giving very fast cooling rates of $10^{5}-10^{6} \mathrm{~K} / \mathrm{s}[2,3]$. It may offer two advantages: (i) thermal annealing used to homogenize the single-phase alloy is no longer needed and (ii) highly textured polycrystalline ribbons with improved properties along a specific direction are produced. Anyway, there is not much information on microstructure formation and the crystal alignment (texture) of the melt-spun ribbons. In this contribution we present the results of electron microscopy, electron backscatter diffraction (EBSD) and $\mathrm{X}$-ray structure analyses of melt-spun $\mathrm{Co}_{2} \mathrm{MnAl}$ type HA and will show its growth-related microstructure, chemical micro-inhomogeneity, disordered crystal structure and some specifics of $\{111\}$ fibre texture.

\section{Experimental details}

As-cast pellets of nominal composition, $\mathrm{Co}_{2} \mathrm{MnAl}$ were prepared by arc-melting from pure elements (> 99.9\%) in an Edmund-Buhler MAM1 arc-melter. A ribbon about $1 \mathrm{~mm}$ wide and $40 \mu \mathrm{m}$ thick was produced by melt spinning in a helium atmosphere at the wheel linear speed of $20 \mathrm{~ms}^{-1}$. According to the EDX microanalysis, the chemical composition of the obtained alloy was $\mathrm{Co}_{2.00} \mathrm{Mn}_{1.28} \mathrm{Al}_{1.06}$ with stoichiometry shifted to

*corresponding author; e-mail: piovarci@saske.sk
Mn. The microstructure of the samples was studied by a field-emission gun scanning electron microscope (Tescan Mira-3 FE) equipped with an energy-dispersive Xray analyser (EDX), electron backscattered diffraction (EBSD) acquisition camera, and Channel 5 software. The grain size was measured by image processing. To check phase purity, a two-dimensional diffraction pattern of the $\mathrm{Co}_{2} \mathrm{MnAl}$ melt-spun ribbon was taken in the transmission geometry using a Rigaku D/MAX Rapid II X-ray diffractometer with an image plate detector, Mo $K_{\alpha}$ radiation $(50 \mathrm{kV}, 38 \mathrm{~mA})$ and the collimator diameter of $100 \mu \mathrm{m}$. The measured pattern was consequently converted into one-dimensional intensity versus diffraction angle dependence (Rigaku 2DP software) and processed by standard procedures for the qualitative phase analysis and the Rietveld method crystal structure refinement.

\section{Results and discussion}

The Heusler compounds of the general formula $\mathrm{X}_{2} \mathrm{YZ}$ crystallize in the cubic system $\left(\mathrm{Cu}_{2} \mathrm{MnAl}-L 2_{1}\right.$ structure prototype) [4]. However, the measured diffraction pattern of the studied ribbon, indexed according to the Fm$3 m$ space group, revealed that the intensities of the fcc typical diffractions (e.g. 111, 311,331...) were negligibly small. It indicates that the crystal structure of the investigated sample should be disordered and can be described in the $P m$ - $3 m$ space group (type $B 2$ ). The lattice parameter of the compound determined by the Rietveld method crystal structure refinement using Rigaku PDXL and Fullprof software packages was found to be $0.2889(14) \mathrm{nm}$ (Fig. 1).

EBSD analyses performed on the as-grown ribbon surfaces are presented in Fig. 2 through the standard pole figures. The most grains have their $\langle 111\rangle$ crystal axis nearly perpendicular to the ribbon surface (Fig. 2a). The declination of the $\langle 111\rangle$ axis of about 10 degrees opposite to the direction of spinning can be seen on the pole figure (Fig. 2a). 


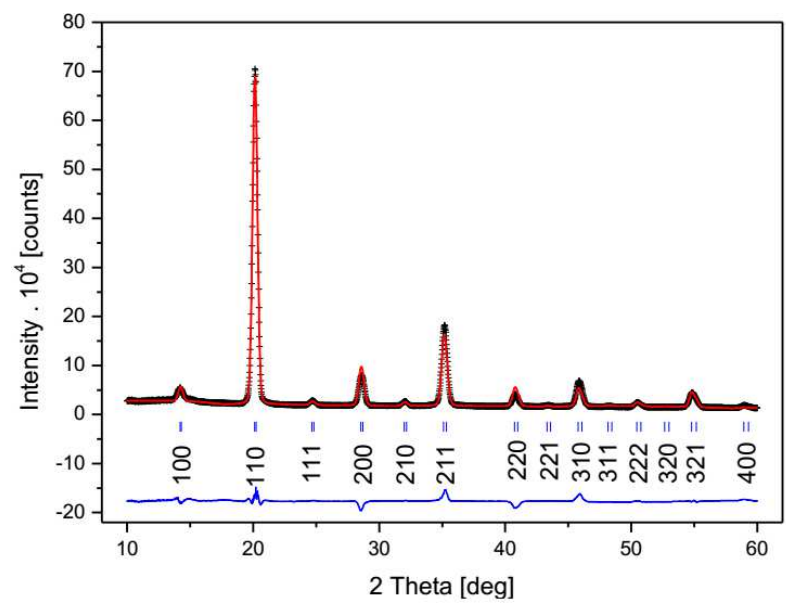

Fig. 1. The Rietveld plot of crystal structure refinement of $\mathrm{Co}_{2} \mathrm{MnAl}$. Vertical markers indicate the position of Bragg peaks.

On the wheel side of the ribbon, the crystal orientation of grains is random which was also confirmed by the pole figure constructed from the EBSD signal (Fig. 2b).

The fracture along the ribbon cross-section shows fine equiaxed grains at the wheel side of the ribbon, follo- wed by the columnar crystals growing perpendicularly to the free surface of the ribbon (Fig. 3a). The cross-section diameter of the columnar crystals is about $6 \mu \mathrm{m}$. The columnar crystals terminate at the free ribbon surface with dendritic morphology (Fig. 3b). Some columnar crystals terminate at the boundary of crystals with dendritic morphology similar. These crystals were obviously nucleated in the melt at the ribbon surface.

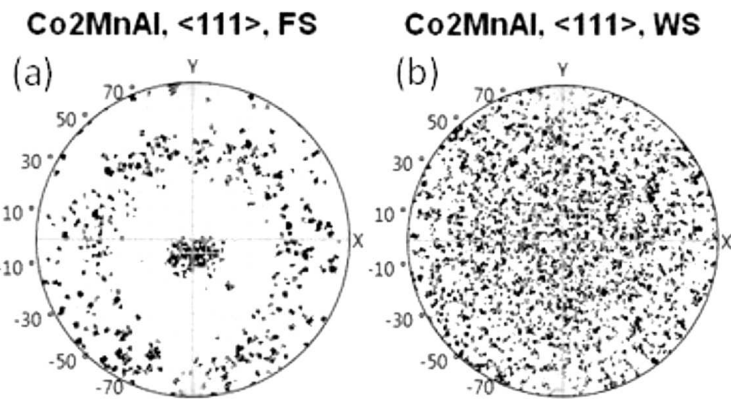

Fig. 2. EBSD analyses on the as-spun ribbon surfaces. (a) The standard pole figure of the ribbon-free surface. (b) The standard pole figure for the ribbon wheel surface.
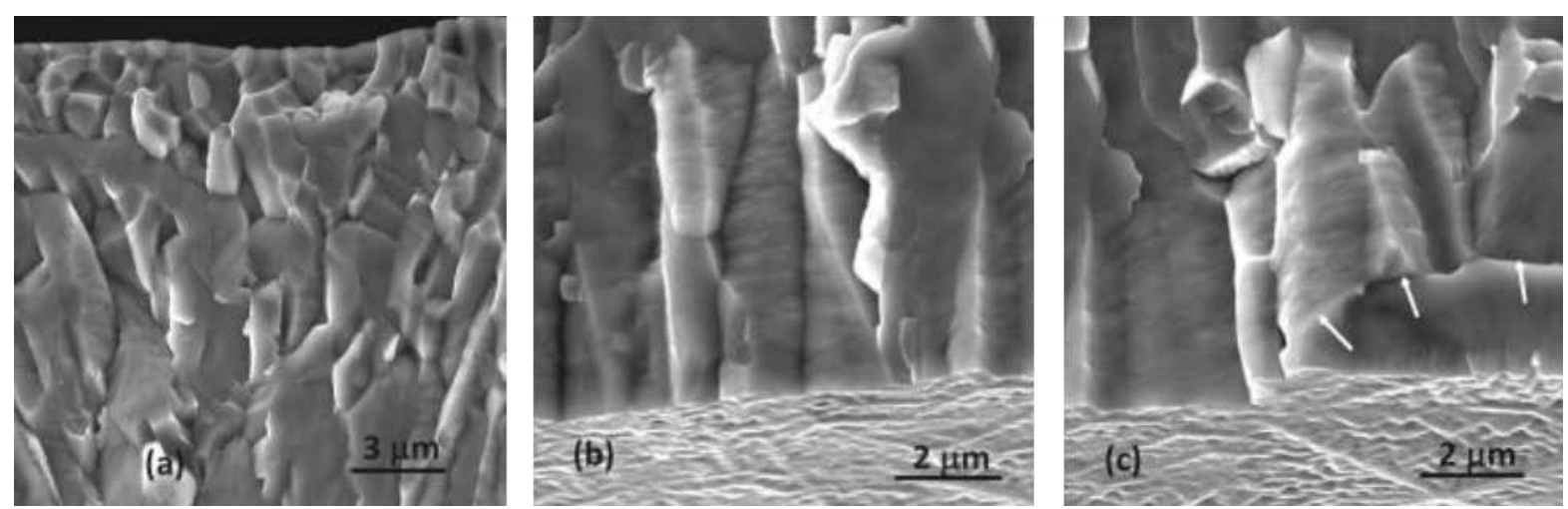

Fig. 3. SEM micrographs of the ribbon fracture along its cross-section. (a) Fine grains at the wheel side followed by columnar crystals. (b) Columnar crystals terminated at the free surface of the ribbon with the morphology of three dendritic arms. (c) Columnar crystals terminated by the grain nucleated at the free surface (arrows).

The smaller crystals nucleated at the wheel side of the ribbon can be seen on the polished cross-section of the ribbon observed in the BSE regime (Fig. 4a). The linear grain size of these small grains is $1.1 \mu \mathrm{m}$. In spite of scratches, the structure of the ribbon can be well-recognised. In the second part of the ribbon, the columnar crystals, declined from the direction perpendicular to the wheel surface, develop. Inside the columnar crystals, some growth structures can be seen with brighter and darker lines in the growth direction. The core of these dendrites is darker and the space between is brighter in the BSE regime, indicating changes in chemical composition. The EDAX line analysis records on the growth structure in columnar crystals along the $2.4 \mu \mathrm{m}$ length (Fig. 4b) clearly show that chemical composition in the part of the melt-spun ribbon grown by dendritic growth is inhomogeneous on the $\mu \mathrm{m}$ scale. The darker parts of the growth structure are enriched in $\mathrm{Al}$ and $\mathrm{Ni}$, while brighter parts are enriched in Mn. This inhomogeneity is in agreement with the contrast observed in the BSE regime. The BSE signal is stronger from the elements with a higher atomic number (atomic numbers: $\mathrm{Al}-13, \mathrm{Mn}-25, \mathrm{Co}-27$ ).

According to the EBSD analysis, the columnar crystals in the studied melt-spun HA have the preferred orientation with the $\{111\}$ planes oriented nearly parallel to the ribbon surface. This type of fibre texture is different from 


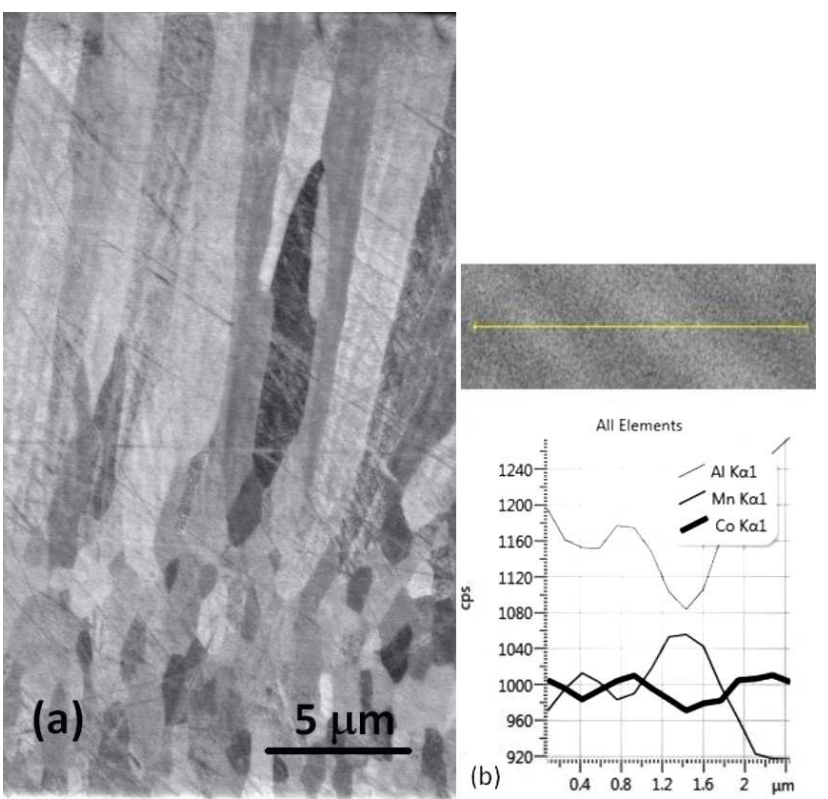

Fig. 4. Ribbon longitudinal section. (a) The contrast in the BSE regime shows fine grains at the wheel side and columnar crystals on the free surface side with an inner structure of growth lines. (b) EDAX line analysis records on the $2.4 \mathrm{\mu m}$ length across growth lines. Darker parts are enriched in $\mathrm{Co}$ and $\mathrm{Al}$ and brighter parts are enriched in Mn (cps: counts per second).

the so-far reported $\{100\}$ fibre texture observed for meltspun ribbons of the $\mathrm{Ni}_{2} \mathrm{MnGa}$ type HA [5]. Observed declination of the $\langle 111\rangle$ crystal direction by about 10 degrees opposite to the spinning direction is not an unusual phenomenon in melt-spun ribbons [6] and is ascribed to the thermal gradient experienced in the melt-spinning process.

At the free surface of the ribbon, where the thermal gradient is lower, some new nucleation (obviously heterogeneous) appears in the melt; therefore, equiaxial grains grow there. These grains prefer a crystal orientation with the $\{100\}$ plane parallel to the ribbon surface which indicates that they were nucleated at the surface of the melt and the interface energy melt/air controlled this process.

The observed inhomogeneity of chemical composition can be related to the dendritic solidification. As the Xray diffraction and SEM analyses show that the sample is a single-phase Heusler alloy, observed segregation of elements during solidification does not lead to the formation of a new phase (besides nanometre-sized particles located at the boundaries of columnar crystals). The occupation of the crystal lattice positions with Co, Mn, and $\mathrm{Al}$ atoms depends on the position in the dendritic arm (centre or edge). This opinion is also supported by an X-ray analysis. In spite of the small area of irradiation (the collimator diameter of $100 \mu \mathrm{m}$ ), the disorder description corresponds to the average composition (the dendrite arm spacing is $0.6 \mu \mathrm{m}$ ) and it is possible that locally various kinds of disorder [4] can also exist. Con- sequently, not only $\mathrm{Mn}$ and Ga atoms are distributed randomly, but also the Co atom distribution varies locally.

\section{Conclusions}

Based on the obtained experimental results, we can conclude that a typical growth-related microstructure developed in the melt-spun samples of the $\mathrm{Co}_{2} \mathrm{MnAl}$ type Heusler alloy. According to texture analyses by EBSD, the fine grain structure at the wheel side of the ribbon exhibits no texture, while columnar grain structure formed on the free surface side exhibits the $\langle 111\rangle$ fibre texture with a declination by about 10 degrees in the opposition to spinning direction.

The melt-spun ribbon exhibits a single-phase disordered Heusler alloy. The dendritic growth of columnar crystals causes inhomogeneity of the chemical composition on the $\mu \mathrm{m}$ scale with a higher $\mathrm{Co}$ and $\mathrm{Al}$ concentration in the centre of dendritic arms and a higher concentration of $\mathrm{Mn}$ at the dendrite arm boundaries. Our analysis shows that the ribbon must be annealed to obtain homogeneous material.

\section{Acknowledgments}

This work was realized within the framework of the projects: Centre of Excellence of Advanced Materials with Nano- and Submicron Structure (ITMS 26220120019), Infrastructure Improving of Centre of Excellence of Advanced Materials with Nano- and Submicron Structure (ITMS 26220120035), New Materials and Technologies for Energetics (ITMS 26220220061), Nanokop (ITMS 26110230061) which are supported by the Operational Programme "Research and Development" financed through the European Regional Development Fund, APVV No. 0330-12, VEGA No. 2/0121/16, VEGA No. VVGS-2013-112 and SAS Centre of Excellence: CFNT MVEP.

\section{References}

[1] T. Marukame, T. Ishikawa, S. Hakamata, K. Matsuda, T. Uemura, M. Yamamoto, Appl. Phys. Lett. 90, 012508 (2007).

[2] N. Dearing, A.G. Jenner, IEEE Trans. Magn. 42, 78 (2006).

[3] J.L. Sánchez Llamazares, T. Sanchez, J.D. Santos, M.J. Pérez, M.L. Sanchez, B. Hernando, Ll. Escoda, J.J. Suñol, R. Varga, Appl. Phys. Lett. 92, 012513 (2008).

[4] S. Wurmehl, M.C.M. Alves, J. Morais, V. Ksenofontov, S.R. Teixeira, G. Machado, G.H. Fecher, C. Felser, J. Phys. D Appl. Phys. 40, 1524 (2007).

[5] T. Goryczka, J. Lelatko, B. Górka-Kostrubiec, P. Ochin, H. Morawiec, Eur. Phys. J. Spec. Top. 158, 131 (2008).

[6] C. Herrera, N.B. de Lima, A.M. Kliauga, A.F. Padilha, Mater. Character. 59, 79 (2008). 